\title{
Utilização de recursos florais por abelhas (Hymenoptera, Apoidea) em uma área de Caatinga (Itatim, Bahia, Brasil)
}

\author{
Cândida Maria Lima Aguiar
}

Departamento de Ciências Biológicas, Universidade Estadual de Feira de Santana. Rodovia BR 116, Km 3, 44031-460 Feira

de Santana, Bahia, Brasil. E-mail: claguiar@uefs.br

\begin{abstract}
The use of floral resources by bees (Hymenoptera, Apoidea) in an area of Caatinga (Itatim, Bahia, Brazil). This study was designed to identify important food resource plants used by bee species in a Caatinga area, as well as describe the local patterns of floral use by bees. A total of 1,145 foraging bees, belonging to 60 species, were captured while visiting 50 plant species. Melochia tomentosa L., Sida galheirensis Ulbr., Erythroxylon catingae P. Cowan, and Ziziphus cotinifolia Reiss. were the most frequently visited plants. Melochia tomentosa, Solanum paniculatum L. and S. galheirensis were visited by larger number of bee species. Some oligolectic bees were identified. Apis mellifera Linnaeus, 1758 and Trigona spinipes (Fabricius, 1793) had the largest trophic niche breadth (2.71 and 2.31). The trophic niche overlap was highest (0.52) between Xylocopa grisescens Lepeletier, 1841 and Frieseomelitta silvestrii (Friese, 1902). The low trophic niche overlap between Apis mellifera and native stingless bees seems to be the result of intensive exploration of only a few flower sources by Africanized bees, not frequently visited by meliponids.
\end{abstract}

KEY WORDS. Bee plants, floral visitors assemblages, semi-arid northeastern Brazil, trophic niche.

A crise da polinização nos países temperados desencadeou um aumento no interesse pela conservação e pela procura de visitantes florais com potencial para uso como polinizadores (KevAN \& IMPERATRIZ-FonSECA 2002). Apesar de o Brasil possuir uma fauna de abelhas diversificada, e portanto um potencial elevado para identificar e manejar espécies nativas com vista à polinização, o pouco conhecimento sobre a diversidade regional e sobre a bionomia das espécies cria uma situação favorável à introdução de polinizadores estrangeiros, para os quais há maior conhecimento bionômico e sobre o seu manejo (Silveira et al. 2002), sem que haja qualquer avaliação das conseqüências ecológicas desta prática. Para o desenvolvimento de programas de conservação e manejo de polinizadores nativos, é imprescindível conhecer as preferências alimentares e o modo como as espécies utilizam os recursos disponíveis, além do conhecimento detalhado da biologia reprodutiva das espécies.

Pouco se sabe sobre os recursos florais necessários à manutenção das comunidades de abelhas em habitats naturais brasileiros. Algumas investigações têm sido conduzidas através da amostragem dos indivíduos nas flores ou da análise do pólen coletado pelas espécies eussociais. Levantamentos da flora visitada pelas abelhas foram realizados em áreas de cerrado (PEDRo \& Camargo 1991, Martins 1995, Albuquerque \& Mendonça 1996, Carvalho \& Bego 1997), floresta Atlântica (Wilms et al. 1996, Wilms \& Wiechers 1997), floresta de altitude (Locatelli \& MAChado 2001), restingas e dunas (Silva \& Martins 1999, Alves-dos-
SANTOS 1999), vegetação xeromórfica do sul do Brasil (SCHLINDWEIN 1998) e caatinga (Aguiar et al. 1995, CARVAlHo \& MarCHini 1999).

Face ao pouco conhecimento disponível sobre a fauna de abelhas e a flora melitófila das regiões semi-áridas do Nordeste do Brasil, o presente trabalho foi desenvolvido com o objetivo de identificar as espécies de plantas utilizadas pelas espécies de abelhas em uma área de caatinga, bem como analisar o modo de utilização dos recursos florais, usando como ferramentas as medidas de amplitude e sobreposição dos nichos tróficos das abelhas.

\section{MATERIAL E MÉTODOS}

Este estudo foi desenvolvido em uma área de Caatinga no município de Itatim, $\left(12^{\circ} 42^{\prime} \mathrm{S}, 39^{\circ} 46^{\prime} \mathrm{W}\right)$, Bahia. As coletas de abelhas e plantas foram realizadas em uma área de Caatinga arbustiva aberta com árvores esparsas, em uma região dominada por formações rochosas do tipo inselbergs. Na base dessas formações há uma vegetação diferenciada, de mata semicaducifólia arbóreo-arbustiva. Uma descrição detalhada da composição florística local pode ser encontrada em FrançA et al. (1997). A região apresenta clima semi-árido tropical, com temperatura média de $24,3^{\circ} \mathrm{C}$, média das mínimas $20,6^{\circ} \mathrm{C}$ e média das máximas $29,9^{\circ} \mathrm{C}$. A precipitação pluviométrica média é de $551 \mathrm{~mm} /$ ano, mas podem ocorrer grandes variações entre anos (142 a $1206 \mathrm{~mm} / \mathrm{ano}$ ) (BAHIA 1994). 
O período de estudo foi de setembro de 1996 a novembro de 1997 e as coletas foram realizadas em intervalos aproximadamente mensais. Em cada amostragem, dois coletores percorriam uma trilha pré-existente, em dois dias (12:00 às 18:00 $\mathrm{h}$ no primeiro dia e 06:00 às 12:00 h no segundo), totalizando 180 horas de amostragem. As abelhas foram capturadas com rede entomológica durante a visita às flores, sem escolha. $\mathrm{O}$ material testemunho (abelhas e plantas) encontra-se depositado na Coleção Entomológica da Universidade Estadual de Feira de Santana (CUFS) e no Herbário da mesma Instituição (HUEFS).

A amplitude do nicho trófico das abelhas foi calculada através do índice de SHANnon (1948) usando a fórmula $\mathrm{H}^{\prime}=-\Sigma \mathrm{p}_{\mathrm{k}} \mathrm{x} \ln \mathrm{p}_{\mathrm{k}^{\prime}}$ onde $\mathrm{p}_{\mathrm{k}}$ é a proporção de indivíduos coletados na planta $K$ e ln é o logarítmo neperiano do valor $p_{k}$. A equitatividade das visitas de cada espécie de abelha às espécies de plantas foi calculada através da fórmula $\mathrm{J}^{\prime}=\mathrm{H}^{\prime} / \mathrm{H}^{\prime}{ }_{\max }$, onde $\mathrm{H}^{\prime}{ }_{\max }$ é o logaritmo neperiano do número total de espécies de plantas visitadas pela espécie de abelha.

A sobreposição dos nichos tróficos das espécies foi calculada pelo índice de Schoener (1968), usando a fórmula $\mathrm{NO}_{\text {ih }}=1-1 / 2 \Sigma_{\mathrm{k}}\left|\mathrm{p}_{\mathrm{ik}}-\mathrm{p}_{\mathrm{hk}}\right|$, onde "i" e " $\mathrm{h}$ " representam as espécies de abelhas comparadas, $\mathrm{p}_{\mathrm{ik}}$ e $\mathrm{p}_{\mathrm{hk}}$ são as proporções de indivíduos respectivamente das espécies de abelha " $\mathrm{i}$ " e " $\mathrm{h}$ " coletados na espécie de planta k. $\mathrm{p}_{\mathrm{ik}}$ é obtida dividindo-se o número de indivíduos da espécie "i"coletado na planta k pelo número total de indíviduos de " $\mathrm{i}$ " obtidos em todas as plantas. Este índice permite analisar a sobreposição entre cada conjunto de duas espécies; as espécies com mais de 20 indivíduos foram combinadas aos pares, e todas as combinações foram analisadas.

\section{RESULTADOS}

Um total de 1.145 indivíduos pertencentes a 60 espécies de abelhas foram coletados, sendo 37 espécies de Apidae, nove de Halictidae, oito de Megachilidae e seis de Colletidae. A maioria das espécies de abelhas foi representada por poucos indivíduos e apenas dez espécies foram representadas por mais de 20 indivíduos. Estas foram analisadas quanto à amplitude e ao grau de sobreposição do nicho trófico.

As abelhas visitaram 50 espécies de plantas (Tab. I). A grande maioria dos indivíduos (79\%) foram capturados em 13 plantas, destacando-se Melochia tomentosa L. (14\%), Sida galheirensis Ulbr. (9\%), Erythroxylon catingae P. Cowan (9\%), Ziziphus cotinifolia Reiss. (8\%) e Acacia bahiensis Benth. (7\%). Considerando a distribuição dos indivíduos visitantes nas famílias botânicas, Malvaceae (15\%), Sterculiaceae (14\%), Caesalpiniaceae (10\%), Erythroxylaceae (9\%), Rhamnaceae (8\%) e Mimosaceae $(7 \%)$ foram mais importantes para as abelhas. As plantas visitadas por maior número de espécies de abelhas foram: M. tomentosa (24 espécies), Solanum paniculatum L. (19), $S$. galheirensis (15), Z. cotinifolia (12), Caesalpinia pyramidalis Tul. (11), Cardiospermum corindum L. (11), Poeppigia procera Presl. (11), Passiflora foetida L. (11) e Chaetocalyx scandens (L.) Urban (9).

Colletidae foi representada por seis espécies (75 indivíduos), que visitaram 11 espécies de plantas (Tab. II). Malvaceae (principalmente $S$. galheirensis e Herissantia crispa (L.) Briz) foi a família botânica mais importante para estas abelhas (79\% dos indivíduos). A concentração do forrageamento em Malvaceae foi evidente para Hylaeus sp. 1 (75\% dos 16 indivíduos), Perditomorpha sp. (92\% de 46) e Sarocolletes fulva Moure \& Urban, 1992 (100\% de 7). Sapindaceae (C. corindum) tam- bém teve importância para Colletidae, tendo sido visitada por cinco espécies e por $8 \%$ dos indivíduos.

As oito espécies de Megachilidae (22 indivíduos) visitaram 10 espécies de plantas (Tab. II). Caesalpiniaceae (P. procera), Fabaceae (C. scandens) e Sterculiaceae (M. tomentosa) receberam visitas de maior número de espécies.

Nove espécies de Halictidae (120 indivíduos) foram coletadas, visitando 26 espécies de plantas (Tab. II). O maior número de espécies foi encontrado nas flores de $S$. paniculatum $(\mathrm{n}=6)$ e de $S$. galheirensis $(\mathrm{n}=4)$. As famílias botânicas mais importantes, em termos de número de visitantes, foram Malvaceae $(28 \%)$, Portulacaceae $(26 \%)$ e Solanaceae $(8 \%)$. A importância das duas primeiras foi influenciada principalmente pelas atividades de forrageamento de Dialictus opacus (Moure, 1940), que embora tenha visitado 16 espécies de plantas, concentrou-se em S. galheirensis e em Portulaca marginata H.B. \& K. ( $21 \%$ dos indivíduos em cada planta).

As 37 espécies de Apidae (928 indivíduos) visitaram 44 espécies de plantas. Para as 25 espécies não-eussociais (Apidae menos Apis, Bombus, Frieseomelitta, Melipona, Partamona, Trigona e Trigonisca), as plantas mais importantes foram M. tomentosa (17 espécies, 26\% dos indivíduos), S. paniculatum (oito espécies, $21 \%$ dos indivíduos) e C. pyramidalis (seis espécies, 15\% dos indivíduos). Oito das dez espécies de Centridini coletadas visitaram M. tomentosa (34\% dos indivíduos), quatro visitaram $S$. paniculatum ( $18 \%$ dos indivíduos) e $P$. procera (12\% dos indivíduos), enquanto para as espécies de Xylocopini, além de $M$. tomentosa (quatro espécies, $25 \%$ dos indivíduos), também $C$. pyramidalis foi importante (duas espécies, $45 \%$ dos indivíduos). As nove espécies de meliponíneos (120 indivíduos) visitaram 19 espécies de plantas, destacando-se dentre estas, $Z$. cotinifolia (seis espécies, 29\% dos indivíduos).

Apis mellifera Linnaeus, 1758 (588 indivíduos) foi a espécie mais freqüente em nove das 13 plantas mais visitadas. Algumas plantas, como A. bahiensis, E. catingae e C. corindum, foram intensivamente visitadas por $A$. mellifera e pouco, ou não visitadas, por outras espécies. A. mellifera visitou o maior número de espécies de plantas (33), sendo que 54\% dos indivíduos foram capturados em apenas 4 espécies: $M$. tomentosa (17\%), E. catingae (16\%), A. bahiensis (12\%) e Z. cotinifolia (9\%).

A abelha africanizada apresentou o nicho trófico mais amplo (2,71), seguida por Trigona spinipes (Fabricius, 1793) $(2,31)$, Pseudaugochlora pandora (Smith, 1853) $(1,97)$ e Dialictus opacus (Moure, 1940) $(1,83)$ (Tab. III). T. spinipes visitou 12 espécies de plantas, todavia nenhuma foi intensivamente visitada, o que é refletido pela alta equitatividade $\left(J^{\prime}=0,93\right)$ das visitas desta espécie, enquanto Frieseomelitta silvestrii (Friese, 1902) foi registrada em cinco plantas, mas concentrou o forrageamento em apenas duas $\left(Z\right.$. cotinifolia e $C$. pyramidalis; $\mathrm{J}^{\prime}=$ $0,64)$. Dentre as espécies com nicho mais estreito $(0,96)$ estão Diadasina riparia (Ducke, 1908) e Perditomorpha sp., que concentraram o forrageio em espécies de Malvaceae. Exomalopsis analis Spinola, 1853, embora tenha apresentado um valor intermediário $(1,39)$ para o nicho trófico, teve $70 \%$ dos indivíduos coletados em $S$. paniculatum.

O maior valor absoluto de sobreposição de nicho trófico $(0,52)$ foi encontrado entre Xylocopa grisescens Lepeletier, 1841 e F. silvestrii (Tab. IV). A maioria dos indivíduos destas duas espécies, registradas em um número relativamente pequeno de plantas, foram coletados em C. pyramidalis. Outros dois pares de espécies apresentaram sobreposição acima de 0,4: Centris 
Tabela I. Número de indivíduos e de espécies de abelhas coletado por planta, em Itatim, Bahia.

\begin{tabular}{|c|c|c|c|}
\hline Famílias & Espécies de plantas & Número de espécies de abelhas & Número de indivíduos \\
\hline Alismataceae & Echinodorus subulatus Griseb & 1 & 1 \\
\hline Amaranthaceae & Gomphrena holosericea Moq. & 1 & 1 \\
\hline \multirow[t]{2}{*}{ Anacardiaceae } & Anacardium occidentale L. & 2 & 9 \\
\hline & Spondias tuberosa Arruda $\left(^{*}\right)$ & 1 & 1 \\
\hline Arecaceae & Syagrus vagans (Bondar) A.D. Hawkes. $\left(^{*}\right)$ & 1 & 1 \\
\hline Bignoniaceae & Tabebuia heptaphylla (Vell.) Toledo & 4 & 6 \\
\hline \multirow[t]{2}{*}{ Boraginaceae } & Cordia aff. globosa (Jacq.) H.B. \& K. $\left(^{\star}\right)$ & 2 & 19 \\
\hline & Cordia latiloba I.M. Johnston & 3 & 11 \\
\hline \multirow[t]{2}{*}{ Cactaceae } & Cereus peruvianus (L.) Mill. & 1 & 3 \\
\hline & Opuntia palmadora Britton \& Rose $\left(^{*}\right)$ & 3 & 23 \\
\hline \multirow[t]{5}{*}{ Caesalpiniaceae } & Caesalpinia pyramidalis Tul $\left(^{*}\right)$ & 11 & 61 \\
\hline & Chamaecrista belemii (I. \& B.) I. \& B. $\left(^{*}\right)$ & 3 & 11 \\
\hline & Peltogyne pauciflora Benth. & 4 & 5 \\
\hline & Poeppigia procera Presl. & 11 & 26 \\
\hline & Senna spectabilis (DC.) I. \& B. & 7 & 13 \\
\hline \multirow[t]{2}{*}{ Capparaceae } & Capparis jacobinae Moric. $\left(^{*}\right)$ & 1 & 1 \\
\hline & Capparis yco (Mart.) Eichl. $\left(^{\star}\right)$ & 7 & 38 \\
\hline \multirow[t]{2}{*}{ Convolvulaceae } & Evolvulus glomeratus Nees \& Mart. & 2 & 7 \\
\hline & Jacquemontia sp. & 2 & 2 \\
\hline Erythroxylaceae & Erythroxylon catingae Plowman & 3 & 101 \\
\hline \multirow[t]{3}{*}{ Euphorbiaceae } & Croton echioides Baill. & 1 & 2 \\
\hline & Croton moritibensis Baill. & 1 & 1 \\
\hline & Jatropha mollissima Baill. $\left(^{*}\right)$ & 1 & 5 \\
\hline Fabaceae & Chaetocalyx scandens (L.) Urban & 9 & 17 \\
\hline Lamiaceae & Rhaphiodon echinus (Nees. \& Mart.) Schrad & 2 & 3 \\
\hline Limnocharitaceae & Hydrocleis nymphaeoides Buch. & 2 & 4 \\
\hline Malpighiaceae & Stigmaphyllon auriculatum (Cav.) A. Juss. & 2 & 4 \\
\hline \multirow[t]{7}{*}{ Malvaceae } & Herissantia crispa (L.) Briz. $\left(^{*}\right)$ & 7 & 59 \\
\hline & Herissantia tiubae (K. Sch.) Briz. $\left(^{\star}\right)$ & 2 & 5 \\
\hline & Pavonia cancellata (L.) Cav. & 1 & 1 \\
\hline & Sida cordifolia $\mathrm{L}$. & 1 & 1 \\
\hline & Sida galheirensis Ulbr. $\left({ }^{*}\right)$ & 15 & 102 \\
\hline & Sida sp. & 3 & 4 \\
\hline & Sidastrum paniculatum (L.) Fryxell & 3 & 3 \\
\hline Menyanthaceae & Nymphoides indica (L.) Kuntze & 1 & 4 \\
\hline \multirow[t]{2}{*}{ Mimosaceae } & Acacia bahiensis Benth. & 3 & 78 \\
\hline & Mimosa arenosa (Willd.) Poir. & 1 & 6 \\
\hline Moraceae & Chlorophora tinctoria (L.) Gaud. & 2 & 12 \\
\hline Myrtaceae & Eugenia rosea DC & 8 & 33 \\
\hline
\end{tabular}


Tabela I. Continuação.

\begin{tabular}{llrr}
\hline \multicolumn{1}{c}{ Famílias } & \multicolumn{1}{c}{ Espécies de plantas } & Número de espécies de abelhas & Número de indivíduos \\
\hline Oxalidaceae & Oxalis sp. & 1 & 2 \\
Passifloraceae & Passiflora cincinnata Mast. & 1 & 1 \\
& Passiflora foetida L. & 11 & 47 \\
Polygalaceae & Polygala sp. & 1 & 1 \\
Portulacaceae & Portulaca marginata H.B. \& K. & 8 & 36 \\
Rhamnaceae & Ziziphus cotinifolia Reiss. (*) & 12 & 98 \\
Sapindaceae & Cardiospermum corindum L. & 11 & 47 \\
Sapotaceae & Sideroxylon obtusifolium (R. \& S.) Penn. & 3 & 4 \\
Solanaceae & Solanum paniculatum L. & 19 & 39 \\
Sterculiaceae & Solanum gardnerii Sendtn. & 2 & 3 \\
\hline
\end{tabular}

* Espécies endêmicas da caatinga, segundo GIULIETTI et al. (2002).

aenea Lepeletier, 1841, E. analis e Perditomorpha sp., D. opacus. Por outro lado, Perditomorpa sp. apresentou em geral os menores valores de sobreposição de nicho trófico com as outras espécies. A sobreposição do nicho trófico entre A. mellifera e as outras abelhas variou de 0 a 0,4 , sendo que a maior sobreposição ocorreu com D. riparia.

\section{DISCUSSÃO}

Das 50 espécies de plantas visitadas, aproximadamente 14 (28\%) espécies representaram recursos florais de maior importância para a comunidade de abelhas estudada, 13 delas por serem visitadas por muitos indivíduos ( $\mathrm{n}>30$; Tab. I), oito por serem visitadas por muitas espécies $(n>10)$ de abelhas e sete (M. tomentosa, S. galheirensis, Z. cotinifolia, S. paniculatum, $P$. foetida, C. corindum e C. pyramidalis) por ambos os aspectos. Adicionalmente, outras 6 espécies vegetais receberam visitas de um número moderado ( 7 a 9) de espécies de abelhas. Outras plantas pouco visitadas podem ser fundamentais para a manutenção da diversidade de abelhas especialistas. Tanto espécies endêmicas (como S. galheirensis, Z. cotinifolia e C. pyramidalis) (Giulietri et al. 2002), quanto espécies ruderais (como $M$. tomentosa e $S$. paniculatum) foram importantes para as abelhas.

As proporções de indivíduos visitantes nas diferentes famílias de plantas em Itatim diferiram bastante da observada na caatinga de São João do Cariri (Paraíba) (Aguiar et al. 1995) e em outros tipos de vegetação. Sterculiaceae, Erythroxylaceae e Rhamnaceae foram importantes apenas em Itatim, enquanto Bromeliaceae, Cactaceae e Convolvulaceae o foram em São João do Cariri. Apenas Caesalpiniaceae e Malvaceae foram importantes para as abelhas em ambas as áreas, sendo $C$. pyramidalis e $S$. galheirensis as espécies mais visitadas destas duas famílias, nas duas áreas.

Dentre as espécies de abelhas mais freqüentemente capturadas, duas constituem casos conspícuos de oligolectia ( $D$. riparia e Perditomorpha sp., associadas a Malvaceae). Embora o pequeno número de indivíduos amostrados impossibilite uma análise segura do modo de utilização de recursos por várias espécies de abelhas, é possível que haja outras espécies oligolécticas, como parece ser o caso de Hylaeus sp. 1 e $S$. fulva. A associação de Colletidae com Malvaceae tem sido registrada na literatura e várias espécies oligolécticas desta família (incluindo duas de Perditomorpha), assim como D. riparia (Apidae) foram registradas também em outra vegetação xeromórfica, no sul do Brasil (SCHLINDWEIN 1998).

Nas três espécies eussociais de Apidae (A. mellifera, $T$. spinipes e $F$. silvestrii) e nas duas espécies de Halictidae (D. opacus e $P$. pandora) analisadas, o padrão de uso dos recursos foi generalista (politrófico), apesar de algumas espécies terem concentrado o forrageio em determinadas plantas. D. opacus apresentou nicho trófico amplo em Itatim, semelhante ao observado para várias outras espécies do gênero Dialictus, consideradas polilécticas (SCHLINDWEIN 1998).

A maior amplitude do nicho trófico entre as abelhas na caatinga de Itatim foi registrada para A. mellifera, o que também ocorreu em áreas de cerrado (MARTINs 1995), floresta atlântica (Wilms et al. 1996) e restinga (Silva \& MarTins 1999), onde T. spinipes também foi uma das espécies com nicho trófico mais amplo. As análises de pólen têm confirmado o alto grau de polilectia destas duas espécies, similares em seus hábitos generalistas e oportunistas, caracterizadas por explorar intensivamente os recursos mais abundantes (CorTOPASSI-LAURINO \& RAMALHO 1988).

A maioria dos pares de espécies analisados apresentaram sobreposição do nicho trófico baixa a moderada, inferior a 0,3. Isto pode estar relacionado com diferentes graus de preferência por plantas distintas (WILMs et al. 1996), mas outros fatores como diferenças entre a extensão do período de florescimento e as atividades sazonais das abelhas solitárias, também podem ter um papel relevante na separação dos nichos tróficos. Um número pequeno de pares de espécies teve sobreposição acima de 0,4, semelhante ao observado por WiLms et al. (1996) na floresta atlântica. Observa-se que nos três pares de espécies com maior sobreposição em Itatim $(X$. grisescens /F. silvestrii; $C$. aenea / E. analis e Perditomorpha sp. / D. opacus, os tamanhos corporais, e conseqüentemente a biomassa, são bastante diferentes, 
Tabela II. Espécies de abelhas e plantas visitadas em Itatim, Bahia. O número de indivíduos coletado em cada espécie de planta é apresentado em negrito, entre parênteses.

\begin{tabular}{|c|c|c|}
\hline \multirow{2}{*}{ Abelhas } & \multicolumn{2}{|r|}{ Plantas visitadas } \\
\hline & Famílias & Espécies \\
\hline \multicolumn{3}{|l|}{ Hylaeinae } \\
\hline \multirow[t]{4}{*}{ Hylaeus sp. 1} & Caesalpiniaceae & Caesalpinia pyramidalis (1) \\
\hline & Malvaceae & Sida galheirensis (12) \\
\hline & Portulacaceae & Portulaca marginata (1) \\
\hline & Sapindaceae & Cardiospermum corindum (2) \\
\hline Hylaeus sp. 2 & Sapindaceae & C. corindum (1) \\
\hline \multicolumn{3}{|l|}{ Colletinae, Colletini } \\
\hline \multirow[t]{3}{*}{ Colletes sp. } & Sapindaceae & C. corindum (1) \\
\hline & Myrtaceae & Eugenia rosea (1) \\
\hline & Solanaceae & Solanum paniculatum (1) \\
\hline \multicolumn{3}{|l|}{ Paracolletini } \\
\hline \multirow[t]{4}{*}{ Perditomorpha sp. } & Euphorbiaceae & Croton moritibensis (1) \\
\hline & Malvaceae & S. galheirensis (30), Herissantia crispa (12), Pavonia cancellata (1) \\
\hline & Portulacaceae & P. marginata (1) \\
\hline & Sapindaceae & C. corindum (1) \\
\hline Protodiscelis palpalis (Ducke, 1909) & Limnocharitaceae & Hydrocleis nymphaeoides (2) \\
\hline Sarocolletes fulva Moure \& Urban 1992 & Malvaceae & H. crispa (3), Sida sp. (2), S. galheirensis (2) \\
\hline \multicolumn{3}{|l|}{ Halictidae } \\
\hline \multicolumn{3}{|l|}{ Augochlorini } \\
\hline \multirow[t]{2}{*}{ Augochlora thalia Smith, 1879} & Portulacaceae & P. marginata (1) \\
\hline & Sapotaceae & Sideroxylon obtusifolium (1) \\
\hline \multirow[t]{4}{*}{ Augochlora (Augochlora) sp. 1} & Convolvulaceae & Jacquemontia sp. (1) \\
\hline & Malvaceae & H. crispa (1), S. galheirensis (2) \\
\hline & Passifloraceae & Passiflora foetida (1) \\
\hline & Portulacaceae & P. marginata (5) \\
\hline Augochlora (Augochlora) sp. 2 & Malvaceae & S. galheirensis (1) \\
\hline Augochloropsis callichroa (Cockerell, 1900) & Solanaceae & S. paniculatum (1) \\
\hline Augochloropsis sp. 1 & Solanaceae & S. paniculatum (1) \\
\hline Augochloropsis sp. 2 & Solanaceae & S. paniculatum (1) \\
\hline \multirow[t]{6}{*}{ Pseudaugochlora pandora (Smith, 1853) } & Boraginaceae & Cordia latiloba (2) \\
\hline & Caesalpiniaceae & C. pyramidalis (4), Poeppigia procera (3), Senna spectabilis (4) \\
\hline & Capparaceae & Capparis yсо (4) \\
\hline & Fabaceae & Chaetocalyx scandens (1) \\
\hline & Solanaceae & S. paniculatum (3) \\
\hline & Sterculiaceae & Melochia tomentosa (1) \\
\hline \multicolumn{3}{|l|}{ Halictini } \\
\hline \multirow[t]{6}{*}{ Dialictus opacus (Moure, 1940) } & Anacardiaceae & Spondias tuberosa (1) \\
\hline & Boraginaceae & Cordia aff. globosa (1) \\
\hline & Caesalpiniaceae & P. procera $(1)$ \\
\hline & Fabaceae & C. scandens (1) \\
\hline & Lamiaceae & Rhaphiodon echinus (2) \\
\hline & Malvaceae & H. crispa (2), S. galheirensis (25) \\
\hline
\end{tabular}


Tabela II. Continuação.

\begin{tabular}{|c|c|c|}
\hline \multirow{2}{*}{ Abelhas } & \multicolumn{2}{|r|}{ Plantas visitadas } \\
\hline & Famílias & Espécies \\
\hline & Myrtaceae & Eugenia rosea (1) \\
\hline & Oxalidaceae & Oxalis sp. (2) \\
\hline & Passifloraceae & P. foetida (1) \\
\hline & Polygalaceae & Polygala sp. (1) \\
\hline & Portulacaceae & P. marginata (25) \\
\hline & Rhamnaceae & Ziziphus cotinifolia (2) \\
\hline & Sapindaceae & C. corindum (4) \\
\hline & Solanaceae & Solanum gardnerii (1), S. paniculatum (1) \\
\hline \multirow[t]{6}{*}{ Dialictus sp. } & Arecaceae & Syagrus vagans (1) \\
\hline & Convolvulaceae & Evolvulus glomeratus (1) \\
\hline & Erythroxylaceae & Erythroxylon catingae (4) \\
\hline & Malvaceae & H. crispa (2), S. galheirensis (1) \\
\hline & Sapotaceae & S. obtusifolium (1) \\
\hline & Solanaceae & S. paniculatum (1) \\
\hline \multicolumn{3}{|l|}{ Megachilidae } \\
\hline \multicolumn{3}{|l|}{ Anthidiini } \\
\hline Dicranthidium arenarium (Ducke, 1907) & Sapindaceae & C. corindum (1) \\
\hline \multirow[t]{3}{*}{ Dicranthidium luciae Urban, 1992} & Malvaceae & S. galheirensis (1) \\
\hline & Rhamnaceae & Z. cotinifolia (3) \\
\hline & Sterculiaceae & M. tomentosa (1) \\
\hline \multicolumn{3}{|l|}{ Megachilini } \\
\hline \multirow[t]{6}{*}{ Megachile lissotate Moure, 1943} & Bignoniaceae & Tabebuia heptaphylla (2) \\
\hline & Cactaceae & Opuntia palmadora (1) \\
\hline & Fabaceae & C. scandens (1) \\
\hline & Malvaceae & S. galheirensis (1) \\
\hline & Passifloraceae & P. foetida (1) \\
\hline & Sterculiaceae & M. tomentosa (1) \\
\hline \multirow[t]{3}{*}{ Megachile dentipes Vachal, 1909} & Caesalpiniaceae & P. procera (2) \\
\hline & Fabaceae & C. scandens (1) \\
\hline & Sterculiaceae & M. tomentosa (1) \\
\hline Megachile paulistana Schrottky, 1902 & Fabaceae & C. scandens (1) \\
\hline Megachile (Holcomegachile) sp. 1 & Caesalpiniaceae & P. procera (1) \\
\hline Megachile (Leptorachina) sp. 2 & Rhamnaceae & Z. cotinifolia (1) \\
\hline \multirow[t]{2}{*}{ Megachile (cf. Schrottkyapis) sp. 3} & Caesalpiniaceae & P. procera (1) \\
\hline & Myrtaceae & E. rosea $(1)$ \\
\hline \multicolumn{3}{|l|}{ Apidae } \\
\hline \multicolumn{3}{|l|}{ Apini } \\
\hline \multirow[t]{4}{*}{ Apis mellifera Linnaeus, 1758} & Anacardiaceae & Anacardium occidentale (5) \\
\hline & Boraginaceae & C. globosa (18), C. latiloba (8) \\
\hline & Cactaceae & O. palmadora (18) \\
\hline & Caesalpiniaceae & $\begin{array}{l}\text { C. pyramidalis (1), Chamaecrista belemii (8), P. procera (6), } \\
\text { Peltogyne pauciflora (2) }\end{array}$ \\
\hline
\end{tabular}

Continua

Revista Brasileira de Zoologia 20 (3): 457-467, setembro 2003 
Tabela II. Continuação.

\begin{tabular}{|c|c|c|}
\hline \multirow{2}{*}{ Abelhas } & \multicolumn{2}{|r|}{ Plantas visitadas } \\
\hline & Famílias & Espécies \\
\hline \multirow[t]{18}{*}{ Apis mellifera Linnaeus, 1758 (continuação) } & Capparaceae & Capparis jacobinae (1), C. yсо (21) \\
\hline & Convolvulaceae & E. glomeratus (6) \\
\hline & Erythroxylaceae & E. catingae (96) \\
\hline & Euphorbiaceae & Croton echioides (2), Jatropha mollissima (5) \\
\hline & Fabaceae & C. scandens (9) \\
\hline & Lamiaceae & R. echinus (1) \\
\hline & Limnocharitaceae & H. nymphaeoides (2) \\
\hline & Malvaceae & $\begin{array}{l}\text { Herissantia tiubae (1), H. crispa (32), S. galheirensis (11), } \\
\text { Sidastrum paniculatum (1) }\end{array}$ \\
\hline & Menyanthaceae & Nymphoides indica (4) \\
\hline & Mimosaceae & Acacia bahiensis (76), Mimosa arenosa (6) \\
\hline & Moraceae & Chlorophora tinctoria (4) \\
\hline & Myrtaceae & E. rosea $(20)$ \\
\hline & Passifloraceae & P. foetida (31) \\
\hline & Rhamnaceae & Z. cotinifolia (55) \\
\hline & Sapindaceae & C. corindum (32) \\
\hline & Sapotaceae & S. obtusifolium (2) \\
\hline & Solanaceae & S. paniculatum (1), S. gardnerii (2) \\
\hline & Sterculiaceae & M. tomentosa (101) \\
\hline Bombus brevivillus Franklin, 1913 & Sterculiaceae & M. tomentosa (1) \\
\hline Bombus morio Swederus, 1787 & Sterculiaceae & M. tomentosa (1) \\
\hline Eulaema nigrita Lepeletier, 1841 & Boraginaceae & C. latiloba (1) \\
\hline \multirow[t]{4}{*}{ Frieseomelitta silvestrii (Friese, 1902) } & Caesalpiniaceae & C. pyramidalis (18), P. pauciflora (1), S. spectabilis (1) \\
\hline & Capparaceae & C. $y c o(2)$ \\
\hline & Myrtaceae & E. rosea $(1)$ \\
\hline & Rhamnaceae & Z. cotinifolia (19) \\
\hline \multirow[t]{4}{*}{ Melipona asilvai Moure, 1971} & Malvaceae & S. galheirensis (4) \\
\hline & Rhamnaceae & Z. cotinifolia (1) \\
\hline & Sapindaceae & C. corindum (1) \\
\hline & Solanaceae & S. paniculatum (1) \\
\hline Melipona quadrifasciata anthidioides Lepeletier, 1836 & Solanaceae & S. paniculatum (1) \\
\hline \multirow[t]{2}{*}{ Partamona sp. } & Malvaceae & S. paniculatum (1) \\
\hline & Rhamnaceae & Z. cotinifolia (3) \\
\hline \multirow[t]{5}{*}{ Plebeia sp. } & Caesalpiniaceae & S. spectabilis (1) \\
\hline & Mimosaceae & A. bahiensis (1) \\
\hline & Myrtaceae & E. rosea (5) \\
\hline & Rhamnaceae & Z. cotinifolia (1) \\
\hline & Solanaceae & S. paniculatum (1) \\
\hline Trigona gr. fuscipennis Friese, 1900 & Portulacaceae & P. marginata (1) \\
\hline \multirow[t]{2}{*}{ Trigona spinipes (Fabricius, 1793) } & Alismataceae & Echinodorus subulatus (1) \\
\hline & Anacardiaceae & A. occidentale (4) \\
\hline
\end{tabular}

Continua

Revista Brasileira de Zoologia 20 (3): 457-467, setembro 2003 
Tabela II. Continuação.

\begin{tabular}{|c|c|c|}
\hline \multirow{2}{*}{ Abelhas } & \multicolumn{2}{|r|}{ Plantas visitadas } \\
\hline & Famílias & Espécies \\
\hline \multirow[t]{8}{*}{ Trigona spinipes (Fabricius, 1793) (continuação) } & Bignoniaceae & T. heptaphylla (2) \\
\hline & Cactaceae & Cereus peruvianus (3), O. palmadora (4) \\
\hline & Caesalpiniaceae & C. pyramidalis (4), P. pauciflora (1) \\
\hline & Capparaceae & C. $y c o(8)$ \\
\hline & Moraceae & C. tinctoria (8) \\
\hline & Passifloraceae & P. foetida (3) \\
\hline & Rhamnaceae & Z. cotinifolia (4) \\
\hline & Sapindaceae & C. corindum (2) \\
\hline \multirow[t]{4}{*}{ Trigonisca intermedia Moure, 1989} & Caesalpiniaceae & P. pauciflora (1), S. spectabilis (1) \\
\hline & Capparaceae & C. $y \operatorname{co~(1)~}$ \\
\hline & Myrtaceae & E. rosea $(1)$ \\
\hline & Portulacaceae & P. marginata (1) \\
\hline Trigonisca sp. & Rhamnaceae & Z. cotinifolia (7) \\
\hline \multicolumn{3}{|l|}{ Centridini } \\
\hline \multirow[t]{5}{*}{ Centris aenea Lepeletier, 1841} & Caesalpiniaceae & C. pyramidalis (2), P. procera (4) \\
\hline & Passifloraceae & P. foetida (2) \\
\hline & Sapindaceae & C. corindum (1) \\
\hline & Solanaceae & S. paniculatum (9) \\
\hline & Sterculiaceae & M. tomentosa (11) \\
\hline \multirow[t]{3}{*}{ Centris caxiensis Ducke, 1907} & Caesalpiniaceae & C. belemii (1) \\
\hline & Fabaceae & C. scandens (1) \\
\hline & Sterculiaceae & M. tomentosa (4) \\
\hline \multirow[t]{4}{*}{ Centris fuscata Lepeletier, 1841} & Caesalpiniaceae & C. pyramidalis (4), P. procera (3), C. belemii (2) \\
\hline & Passifloraceae & Passiflora cincinnata (1), P. foetida (1) \\
\hline & Solanaceae & S. paniculatum (3) \\
\hline & Sterculiaceae & M. tomentosa (3) \\
\hline Centris leprieuri (Spinola, 1841) & Sterculiaceae & M. tomentosa (1) \\
\hline \multirow[t]{2}{*}{ Centris moerens (Perty, 1833) } & Caesalpiniaceae & S. spectabilis (3) \\
\hline & Malpighiaceae & Stigmaphyllon auriculatum (3) \\
\hline \multirow[t]{2}{*}{ Centris cf. spilopoda Moure, 1969} & Bignoniaceae & T. heptaphylla (1) \\
\hline & Malpighiaceae & S. auriculatum (1) \\
\hline \multirow[t]{2}{*}{ Centris sponsa Smith, 1854} & Solanaceae & S. paniculatum (1) \\
\hline & Sterculiaceae & M. tomentosa (1) \\
\hline \multirow[t]{4}{*}{ Centris tarsata Smith, 1874} & Bignoniaceae & T. heptaphylla (1) \\
\hline & Caesalpiniaceae & C. pyramidalis (1), P. procera (2) \\
\hline & Passifloraceae & P. foetida (3) \\
\hline & Sterculiaceae & M. tomentosa (5) \\
\hline \multirow[t]{6}{*}{ Centris trigonoides Lepeletier, 1841} & Caesalpiniaceae & P. procera (2) \\
\hline & Fabaceae & C. scandens (1) \\
\hline & Passifloraceae & P. foetida (1) \\
\hline & Sapindaceae & C. corindum (1) \\
\hline & Solanaceae & S. paniculatum (3) \\
\hline & Sterculiaceae & M. tomentosa (4) \\
\hline Centris xanthomelaena Moure \& Castro, 2001 & Sterculiaceae & M. tomentosa (1) \\
\hline
\end{tabular}


Tabela II. Continuação.

\begin{tabular}{|c|c|c|}
\hline \multirow{2}{*}{ Abelhas } & \multicolumn{2}{|r|}{ Plantas visitadas } \\
\hline & Famílias & Espécies \\
\hline \multicolumn{3}{|l|}{ Emphorini } \\
\hline \multirow[t]{2}{*}{ Diadasia sp. } & Malvaceae & S. galheirensis (2) \\
\hline & Sterculiaceae & M. tomentosa (1) \\
\hline \multirow[t]{4}{*}{ Diadasina riparia (Ducke, 1908) } & Amaranthaceae & Gomphrena holosericea (1) \\
\hline & Convolvulaceae & Jacquemontia sp. (1) \\
\hline & Malvaceae & H. tiubae (4), H. crispa (7), S. galheirensis (7) \\
\hline & Sterculiaceae & M. tomentosa (1) \\
\hline \multicolumn{3}{|l|}{ Ericrocidini } \\
\hline Mesocheira bicolor (Fabricius, 1804) & Caesalpiniaceae & C. pyramidalis (1) \\
\hline Mesoplia sp. & Capparaceae & C. $y \operatorname{co~(1)~}$ \\
\hline \multicolumn{3}{|l|}{ Eucerini } \\
\hline Melissodes nigroaenea (Smith, 1854) & Caesalpiniaceae & P. procera (1) \\
\hline Melissoptila uncicornis (Ducke, 1910) & Malvaceae & Sida sp. (1) \\
\hline \multicolumn{3}{|l|}{ Exomalopsini } \\
\hline \multirow[t]{6}{*}{ Exomalopsis analis Spinola, 1853} & Erythroxylaceae & E. catingae (1) \\
\hline & Fabaceae & C. scandens (1) \\
\hline & Malvaceae & Sida cordifolia (1), Sida sp. 1 (1), S. galheirensis (1) \\
\hline & Mimosaceae & A. bahiensis (1) \\
\hline & Solanaceae & S. paniculatum (23) \\
\hline & Sterculiaceae & M. tomentosa (4) \\
\hline \multicolumn{3}{|l|}{ Tapinotaspidini } \\
\hline Tapinotaspoides rufescens (Friese, 1899) & Passifloraceae & P. foetida (2) \\
\hline \multicolumn{3}{|l|}{ Xylocopinae } \\
\hline \multicolumn{3}{|l|}{ Ceratinini } \\
\hline \multirow[t]{4}{*}{ Ceratina maculifrons Smith, 1854} & Malvaceae & S. galheirensis (2) \\
\hline & Portulacaceae & P. marginata (1) \\
\hline & Solanaceae & S. paniculatum (2) \\
\hline & Sterculiaceae & M. tomentosa (5) \\
\hline Ceratinula sp. & Sterculiaceae & M. tomentosa (1) \\
\hline \multicolumn{3}{|l|}{ Xylocopini } \\
\hline \multirow[t]{2}{*}{ Xylocopa cearensis Ducke, 1910} & Passifloraceae & P. foetida (1) \\
\hline & Sterculiaceae & M. tomentosa (5) \\
\hline \multirow[t]{7}{*}{ Xylocopa grisescens Lepeletier, 1841} & Caesalpiniaceae & C. pyramidalis (20), S. spectabilis (1) \\
\hline & Capparaceae & C. $y c o(1)$ \\
\hline & Malvaceae & S. paniculatum (1) \\
\hline & Myrtaceae & E. rosea (3) \\
\hline & Rhamnaceae & Z. cotinifolia (1) \\
\hline & Solanaceae & S. paniculatum (2) \\
\hline & Sterculiaceae & M. tomentosa (6) \\
\hline \multirow[t]{3}{*}{ Xylocopa frontalis (Olivier, 1789) } & Caesalpiniaceae & C. pyramidalis (5), S. spectabilis (2) \\
\hline & Rhamnaceae & Z. cotinifolia (1) \\
\hline & Sterculiaceae & M. tomentosa (1) \\
\hline \multirow[t]{2}{*}{ Xylocopa muscaria (Fabricius, 1775) } & Sterculiaceae & M. tomentosa (2) \\
\hline & Solanaceae & S. paniculatum (3) \\
\hline
\end{tabular}


Tabela III. Amplitude (H') e equitatividade (J') dos nichos tróficos das abelhas em Itatim, Bahia.

\begin{tabular}{lcclcc}
\hline \multicolumn{1}{c}{ Espécies } & $\mathrm{H}^{\prime}$ & $\mathrm{J}^{\prime}$ & \multicolumn{1}{c}{ Espécies } & $\mathrm{H}^{\prime}$ & $\mathrm{J}^{\prime}$ \\
\hline Apis mellifera & 2,71 & 0,78 & Xylocopa grisescens & 1,40 & 0,67 \\
Trigona spinipes & 2,31 & 0,93 & Exomalopsis analis & 1,39 & 0,67 \\
Pseudaugochlora pandora & 1,97 & 0,95 & Frieseomelitta silvestrii & 1,14 & 0,64 \\
Dialictus opacus & 1,83 & 0,66 & Diadasina riparia & 0,96 & 0,54 \\
Centris aenea & 1,49 & 0,83 & Perditomorpha sp. & 0,96 & 0,54 \\
\hline
\end{tabular}

Tabela IV. Sobreposição dos nichos tróficos entre espécies de abelhas em Itatim, Bahia.

\begin{tabular}{|c|c|c|c|c|c|c|c|c|c|}
\hline Espécies & $A m$ & Ts & Fs & $D r$ & $E a$ & $\mathrm{Ca}$ & $\mathrm{Xg}$ & Do & $P p$ \\
\hline Apis mellifera & - & & & & & & & & \\
\hline Trigona spinipes & 0,27 & - & & & & & & & \\
\hline Frieseomelitta silvestrii & 0,15 & 0,25 & - & & & & & & \\
\hline Diadasina riparia & 0,39 & 0,30 & 0,00 & - & & & & & \\
\hline Exomalopsis analis & 0,00 & 0,00 & 0,00 & 0,38 & - & & & & \\
\hline Centris aenea & 0,27 & 0,17 & 0,07 & 0,35 & 0,43 & - & & & \\
\hline Xylocopa grisescens & 0,26 & 0,15 & 0,52 & 0,35 & 0,18 & 0,30 & - & & \\
\hline Dialictus opacus & 0,20 & 0,10 & 0,04 & 0,36 & 0,06 & 0,08 & 0,06 & - & \\
\hline Pseudaugochlora pandora & 0,12 & 0,27 & 0,25 & 0,35 & 0,21 & 0,39 & 0,34 & 0,04 & \\
\hline Perditomorpha sp. & 0,11 & 0,02 & 0,00 & 0,36 & 0,03 & 0,02 & 0,00 & 0,42 & 0,00 \\
\hline
\end{tabular}

o que pode resultar em uma pressão competitiva (sensu WiLMs et al. 1996) alta das espécies maiores sobre as menores, se elas usam os recursos simultaneamente.

A sobreposição de $A$. mellifera com as duas espécies de meliponíneos mais abundantes (T. spinipes e F. silvestrii) foi relativamente baixa $(<0,3)$ em Itatim, o que decorreu, pelo menos em parte, da exploração intensiva pela abelha africanizada de algumas fontes florais não visitadas em alta freqüência pelos meliponíneos. Na floresta atlântica, WiLms et al. (1996) também encontraram um valor moderado de sobreposição do nicho trófico entre $A$. mellifera e $T$. spinipes $(0,33)$, porém esses autores observaram que a pressão competitiva (que leva em conta, além da sobreposição do nicho, a abundância relativa e o peso corporal) da abelha africanizada foi geralmente alta sobre quase todas as espécies de meliponíneos.

Apesar do índice de sobreposição geralmente não ter sido alto, observou-se que a abelha africanizada explorou intensivamente alguns recursos importantes para as abelhas nativas, como Z. cotinifolia, M. tomentosa e H. crispa. É preciso considerar também elevada abundância de $A$. mellifera em relação às outras espécies de abelhas. O número de indivíduos da abelha africanizada foi cerca de 14 vezes maior do que o das espécies mais abundantes de meliponíneos ( $T$. spinipes e $F$. silvestrii), enquanto as outras sete espécies de abelhas sem ferrão foram representadas por menos de 10 indivíduos. Finalmente, é importante considerar que o grau de sobreposição não é constante ao longo do ano. As variações temporais estão associadas a mudanças na intensidade de exploração das fontes florais pelas espécies de abelhas (Cortopassi-Laurino \& Ramalho 1988, WiLms \& Wiechers 1997). Na caatinga, a quantidade de recursos florais disponíveis para as abelhas parece ser reduzida, pelo menos em uma parte da estação seca (Aguiar observação pessoal), de modo que é possível que a pressão competitiva de espécies com alta abundância e nicho trófico amplo aumente nos períodos de escassez de recursos.

As coletas de abelhas em flores podem fornecer bons indícios do grau de sobreposição do nicho trófico, embora este método apresente algumas limitações, especialmente o pequeno número de indivíduos capturados. A análise do pólen e néctar estocado nos ninhos seria uma importante ferramenta para complementar a investigação do uso dos recursos florais na caatinga, onde as capturas de abelhas em flores geralmente resultam em uma amostra muito pequena para permitir inferências sobre competição por recursos florais, mesmo para as espécies eussociais (Martins 1994, Aguiar \& Martins 1997).

\section{AGRADECIMENTOS}

A Gilberto M.M. Santos, Janete J. Resende e Valéria M. Monteiro, pelo auxílio no trabalho de campo. A Flávio França e Efigênia Melo, pela identificação das plantas. A Fernando C.V. Zanella, João M.F. Camargo e Sílvia R.M. Pedro, pela identificação das abelhas. A Márcio Z. Cardoso, pelas sugestões ao manuscrito. 


\section{REFERÊNCIAS BIBLIOGRÁFICAS}

Aguiar, C.M.L; C.F. Martins \& A.C.A. Moura. 1995. Recursos florais utilizados por abelhas (Hymenoptera, Apoidea) em área de caatinga (São João do Cariri, Paraíba). Revista Nordestina de Biologia, João Pessoa, 10 (2): 101-117.

Aguiar, C.M.L. \& C.F. Martins. 1997. Abundância relativa, diversidade e fenologia de abelhas (Hymenoptera, Apoidea) na caatinga, São João do Cariri, Paraíba, Brasil. Iheringia, Série Zoologia, Porto Alegre, (83): 151-163.

Albuquerque, P.M.C. \& J.A.C. MendonçA. 1996. Anthophoridae (Hymenoptera; Apoidea) e flora associada em uma formação de cerrado no município de Barreirinhas, MA, Brasil. Acta Amazonica, Manaus, 26 (1/2): 45-54.

Alves-Dos-Santos, I. 1999. Abelhas e plantas melíferas da mata atlântica, restingas e dunas do litoral norte do estado do Rio Grande do Sul, Brasil. Revista Brasileira de Entomologia, São Paulo, 43: 191-223.

BAHIA (Centro de Estatística e Informação). 1994. Informações básicas dos municípios bahianos: Recôncavo Sul.. Salvador, vol. 8, 761p.

Carvalho, A.M.C. \& L.R. Bego. 1997. Explotation of available resources by bee fauna (Apoidea- Hymenoptera) in the Reserva Ecológica do Panga, Uberlândia, State of Minas Gerais, Brazil. Revista Brasileira de Entomologia, São Paulo, 41 (1): 101-107.

Carvalho, C.A.L. \& L.C. Marchini. 1999. Plantas visitadas por Apis mellifera L. no vale do rio Paraguaçu, Município de Castro Alves, Bahia. Revista Brasileira de Botânica, São Paulo, 22 (Supp. 2): 333-338.

Cortopassi-Laurino, M. \& M. Ramalho. 1988. Pollen harvest by Africanized Apis mellifera and Trigona spinipes in São Paulo: botanical and ecological views. Apidologie, Versailles, 19 (1): 1-24.

França, F.; E. Melo \& C.C. Santos. 1997. Flora de inselbergs da região de Milagres, Bahia, Brasil: I. Caracterização da vegetação e lista de espécies de dois inselbergs. Sitientibus, Feira de Santana, 17: 163-184.

Giulietti, A.M.; R.M. Harley; L.P. Queiroz; M.R. Barbosa; A.L. Bocage Neta \& M.A. Figueiredo. 2002. Espécies endêmicas da Caatinga, p. 103-115. In: E.V.S.B SAmpaIO; A.M. GiulietTI; J. VIrgínio \& C. Gamarra Rojas (Eds). Vegetação e flora da Caatinga. Associação Plantas do Nordeste (APNE), Centro Nordestino de Informação, Recife, 176p.

Kevan, P.G. \& V.L. Imperatriz-Fonseca. 2002. Pollinating bees: the conservation link between agriculture and nature. Brasília, Ministério do Meio Ambiente, 313p.
Locatelli, E. \& I.C. Machado. 2001. Bee diversity and their floral resources in a fragment of a tropical altitudinal wet forest ("Brejos de altitude") in Northeastern Brazil. Acta Horticulturae, Hague, 561: 317- 325.

Martins, C.F. 1994. Comunidade de abelhas (Hym., Apoidea) da caatinga e do cerrado com elementos de campo rupestre do estado da Bahia, Brasil. Revista Nordestina de Biologia, João Pessoa, 9 (2): 225-257.

- 1995. Flora apícola e nichos tróficos de abelhas (Hym., Apoidea) na Chapada Diamantina (Lençóis, BA, Brasil). Revista Nordestina de Biologia, João Pessoa, 10 (2): 119-140.

Pedro, S.R.M. \& J.M.F. Camargo. 1991. Interactions on floral resources between the Africanized honey bee Apis mellilfera L. and the native bee community (Hymenoptera: Apoidea) in a natural "cerrado" ecosystem in southeast Brazil. Apidologie, Versailles, 22: 397-415.

SHANnON, C.E. 1948. The mathematical theory of communication, p. 3-91. In: C.E. Shannon \& W. Weaver (Eds). The mathematical theory of communication. Urbana, University Illinois Press, 117p.

Schlindwein, C. 1998. Frequent oligolecty characterizing a diverse bee-plant community in a xerophytic bushland of subtropical Brazil. Studies on Neotropical Fauna and Environment, Lisse, 33: 46-59.

Schoener, T.W. 1968. The Anolis lizard of Bimini: resource partitioning in a complex fauna. Ecology, Durham, 49 (4): 704-726.

Silva, M.C.M. \& C.F. Martins. 1999. Flora apícola e relações tróficas de abelhas (Hymenoptera: Apoidea) em uma área de restinga (Praia de Intermares, Cabedelo - PB, Brasil). Principia, João Pessoa, 7 (3): 40-51.

Silveira, F.A.; C. Pinheiro-Machado; I. Alves-dos-Santos; A.M.P. Kleinert \& V.L. Imperatriz-Fonseca. 2002. Taxonomic constraints for the conservation and sustainable use of wild pollinators - The brazilian wild bees, p. 41-50. In: P.G. KeVAN \& V.L. Imperatriz-Fonseca (Eds). Pollinating bees: the conservation link between agriculture and nature. Brasília, Ministério do Meio Ambiente, 313p.

Wilms, W.; V.L. Imperatriz-Fonseca \& W. Engels. 1996. Resource partitioning between highly eusocial bees and possible impact of the introduced Africanized honey bee on native stingless bees in the Brazilian Atlantic rainforest. Studies on Neotropical Fauna and Environment, Lisse, 31: 137-151.

WiLMS, W. \& B. Wiechers. 1997. Floral resource partitioning between native Melipona bees and the introduced Africanized honey bee in the Brazilian Atlantic rain forest. Apidologie, Versailles, 28: 339-355.

Recebido em 21.III.2003; aceito em 18.VIII.2003. 